\title{
Leitlinien empfehlen Echinocandine bei Candidose
}

- Die European Society of Clinical Microbiology and Infectious Diseases (ESCMID) wird in Kürze eine neue Leitlinie zu Diagnostik und Management der invasiven Candidose veröffentlichen. In vielen Bereichen rücken dabei die Echinocandine nach vorn. Die neue Leitlinie, an der außer der ESCMID auch noch die europäischen Fachgesellschaften EORTC (Onkologie), ESICM (Intensivmedizin), EBMT (Transplantation) und ECMM (Mykologie) beteiligt waren, wurde in einer vorläufigen Endversion von Prof. Andrew Ullmann, Würzburg, vorgestellt. Sie enthält unter anderem Empfehlung zum Management der invasiven Candidose bei Erwachsenen, Kindern, HIV/ AIDS-Patienten sowie in der Hämatotologie/ Onkologie.
Dass von konventionellem Amphotericin B wegen der damit verbundenen Toxizitäten explizit abgeraten werde, sei ein Kernpunkt der Empfehlungen für die Therapie von erwachsenen Intensivpatienten sei, so Ullmann. Auch liposomales Amphotericin erhalte lediglich eine B-Empfehlung. Demgegenüber wird Echinocandinen wie Micafungin $\left(\right.$ Mycamine ${ }^{\circledR}$ ) in der Leitlinie eine A-IEmpfehlung, die stärkste Empfehlung, gegeben.

Wichtig sei auch die korrekte Dauer derTherapie, betonte Ullmann. Leitliniengemäß behandelt nur, wer ab dem Ende der nachgewiesenen Candidämie noch mindestens 14 Tage weiter therapiert. Die Umsetzung dieser Empfehlung erfordere tägliche Blut- kulturen. Obligat gescreent werden sollte nach möglichen Organschäden, darunter Endokarditis, Augenbeteiligung und Thrombenbildung bei Vorliegen von Implantaten. Eine weitere Indikation für eine systemische Antipilztherapie, die zunehmend an Bedeutung gewinnt, ist die Prophylaxe einer Candida-Infektion nach autologer Stammzelltransplantation. Bei dieser Indikation sei Micafungin in der ESCMID-Leitlinie das einzige Echinocandin mit einer A-I-Empfehlung, so Ullmann. Es wird damit genauso eingestuft wie Fluconazol, was die günstige Studienlage zu Micafungin bei dieser Indikation widerspiegelt. Philipp Grätzel von Grätz

Pressekonferenz "Invasive Candidose - Neue Leitlinien empfehlen Echonocandine" im Rahmen des Kongress der International Society of Human and Animal Mycology (ISHAM 2012), Berlin, 12. Juni 2012

Veranstalter: Astellas, München

\section{mHRPC: Cabazitaxel verlängert Überleben}

— Die Therapie mit Cabazitaxel plus Prednison verbessert das Gesamtüberleben von Patienten mit metastasiertem hormonrefraktärem Prostatakarzinom (mHRPC) signifikant. Damit ist das Tubulin-bindende Taxan eine Option bei Progredienz während oder nach einer Docetaxel-basierten Behandlung. Wie die randomisierte Zulassungsstudie TROPIC [de Bono JB et al., Lancet 2010; 376: 1147-54] zeigte, wurde unter Cabazitaxel
(Jevtana ${ }^{\circledast}$ ) eine klinisch relevante Verlängerung des medianen Gesamtüberlebens auf 15,1 Monate erreicht (im Mitoxantron-Vergleichsarm 12,7 Monate). Das relative Sterberisiko verringerte sich signifikant um 30\%, sagte Dr. David Pfister, Aachen. Zudem wurde eine ebenfalls signifikante Verlängerung des medianen progressionsfreien Überlebens von 2,8 Monaten unter Cabazitaxel gegenüber 1,4 Monaten unter Mitoxantron beobachtet.

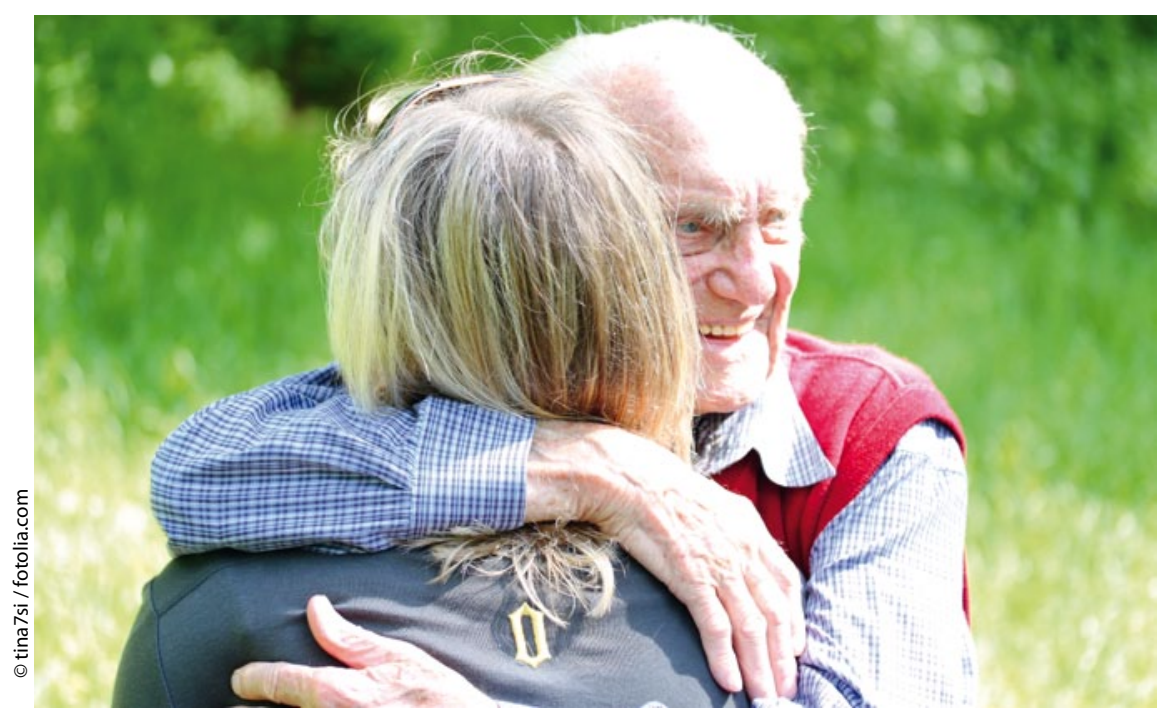

Länger überleben bei weniger Tumorschmerzen, weniger Nebenwirkungen und höherer Lebensqualität - wichtige Faktoren in der Therapie von Prostatakarzinom-Patienten.
Aufgrund der Datenlage bietet sich nach einem Docetaxel-Versagen die Weiterbehandlung mit Cabazitaxel an, ergänzte Prof. Peter Hammerer, Braunschweig. Insbesondere Patienten, die ein rasches Therapieansprechen benötigen, beispielsweise wegen eines schnellen PSA-Anstiegs oder einer ausgeprägten Tumorsymptomatik und drohender Verschlechterung des Allgemeinzustandes, sollten nach Docetaxel-Versagen mit Cabazitaxel weiterbehandelt werden. Erhalten die Patienten Cabazitaxel zu spät im Therapieverlauf bestehe das Risiko, dass sich ihr Allgemeinbefinden so verschlechtert, dass sie nicht mehr chemotherapiefähig sind. Daten aus dem klinischen Alltag [Heidenreich A et al., Eur Urol Suppl 2012; 11; e128] belegen, dass viele Patienten unter der CabazitaxelTherapie nicht nur länger überleben, sondern auch eine höhere Lebensqualität haben. Lediglich 3,6\% entwickelten eine febrile Neutropenie (Grad 3/4). Auch andere schwere Nebenwirkungen (Grad 3/4) waren selten: Eine Neutropenie trat bei 7,2\% und Diarrhöen bei $0,9 \%$ der Patienten auf. Laut Pfister führe die reduzierte Tumorlast dazu, dass die Patienten weniger tumorbedingte Schmerzen haben und folglich weniger Schmerzmedikation benötigten.

Dr. Ralph Hausmann

Fachpresse-Workshop:„3. Expertise Prostata“, Eltville, 19. Juli 2012

Veranstalter: Sanofi-Aventis, Frankfurt/Main 